\title{
Maximum diameter versus volumetric assessment for the response evaluation of vestibular schwannomas receiving stereotactic radiotherapy
}

\author{
Youngmin Choi, MD, PhD', Sungmin Kim, MD¹, Dong-Won Kwak, MS', \\ Hyung-Sik Lee, MD, PhD', Myung-Koo Kang, MD, PhD², Dong-Kun Lee, MD, PhD², \\ Won-Joo Hur, MD, PhD'
}

Departments of ${ }^{1}$ Radiation Oncology, ${ }^{2}$ Otolaryngology-Head and Neck Surgery, Dong-A University College of Medicine, Busan, Korea

Purpose: To explore the feasibility of maximum diameter as a response assessment method for vestibular schwannomas (VS) after stereotactic radiosurgery or fractionated stereotactic radiotherapy (RT), we analyzed the concordance of RT responses between maximum diameters and volumetric measurements.

Materials and Methods: Forty-two patients receiving curative stereotactic radiosurgery or fractionated stereotactic RT for VS were analyzed retrospectively. Twelve patients were excluded: 4 did not receive follow-up magnetic resonance imaging (MRI) scans and 8 had initial MRI scans with a slice thickness $>3 \mathrm{~mm}$. The maximum diameter, tumor volume (TV), and enhanced tumor volume (ETV) were measured in each MRI study. The percent change after RT was evaluated according to the measurement methods and their concordances were calculated with the Pearson correlation. The response classifications were determined by the assessment modalities, and their agreement was analyzed with Cohen kappa statistics.

Results: Median follow-up was 31.0 months (range, 3.5 to 86.5 months), and 90 follow-up MRI studies were analyzed. The percent change of maximum diameter correlated strongly with TV and ETV $(r(p)=0.85,0.63, p=0.000$, respectively). Concordance of responses between the Response Evaluation Criteria in Solid Tumors (RECIST) using the maximum diameters and either TV or ETV were moderate (kappa $=0.58 ; 95 \%$ confidence interval, $0.32-0.85$ ) or fair (kappa $=0.32 ; 95 \%$ confidence interval, $0.05-0.59$ ), respectively.

Conclusions: The percent changes in maximum diameter and the responses in RECIST were significantly concordant with those in the volumetric measurements. Therefore, the maximum diameters can be used for the response evaluation of VS following stereotactic RT.

Keywords: Vestibular schwannoma, Radiotherapy, Response Evaluation Criteria in Solid Tumors (RECIST)

\section{Introduction}

Vestibular schwannomas (VS) are benign, slow-growing tumors. The incidence ranges from 1.1 to 2.21 per 100,000 per year [1-3]. Because of the infrequent occurrence, most studies are retrospective, and their management has several options including observation, surgical resection, or radiotherapy (RT). RT can be relevant due to its non-invasiveness, broad

Received 10 January 2018, Revised 26 April 2018, Accepted 10 May 2018.

Correspondence: Won-Joo Hur, MD, Department of Radiation Oncology, Dong-A University College of Medicine, 26 Daesingongwon-ro, Seo-gu, Busan 49202, Korea. Tel: +82-51-240-2955, E-mail: wjhur@dau.ac.kr

(c) This is an Open Access article distributed under the terms of the Creative Commons Attribution Non-Commercial License (http://creativecommons.org/ licenses/by-nc/4.0/) which permits unrestricted non-commercial use, distribution, and reproduction in any medium, provided the original work is properly cited.

www.e-roj.org 
indications, and high control rates of about 90\% [4-6].

The benign nature of VS precludes invasion into adjacent tissues, so stereotactic RT without margins can be applied with less irradiation to them compared with conventional RT. The tumor control rates of VS following fractionated stereotactic RT were reported to be similar to stereotactic radiosurgery $[7,8]$.

The Response Evaluation Criteria in Solid Tumors (RECIST) are used for malignant tumors, but there is no consensus for a response assessment for a VS following RT. Modern RT plans use three-dimensional target delineation, so volumetric analysis seems to be feasible for response evaluation following RT. The assessment of volumetric response is accurate, but it requires more effort and time compared to RECIST, which just uses the maximal diameter in axial scans. When RECIST produces similar outcomes to the volumetric methods, it can be used confidently.

The effectiveness of RECIST is still unclear for VS receiving stereotactic RT. Therefore, we examined the feasibility of using the linear maximum diameter for the response evaluation of VS after RT. This was done by analyzing the concordance of size changes between maximum diameters and volumes and the agreement of responses between RECIST and volumetric assessments. In addition, we calculated local control rates by the different measurement methods.

\section{Materials and Methods}

\section{Patients}

From December 2010 to April 2016, 42 consecutive VS patients who received stereotactic RT at Dong-A University Hospital were enrolled. Their records were retrospectively reviewed after approval of the Institutional Review Board (No. DAUHIRB-17-083).

Excluding 12 patients who did not have follow-up magnetic resonance imaging (MRI) $(n=4)$ or had MRI slice thickness exceeding $3 \mathrm{~mm}(\mathrm{n}=8), 30$ patients were included in this study. The slice thickness of $2 \mathrm{~mm}$ was recommended for target localization with MRI, but thickness up to $3 \mathrm{~mm}$ was allowed in this study.

\section{Radiotherapy}

Planning computed tomography (CT) was taken with a slice thickness of $2 \mathrm{~mm}$ after immobilizing the patient's head using an individual thermoplastic stereotactic mask (Brainlab, Feldkirchen, Germany). CT data were fused with T1-weighted axial post-contrast MRI scans on iPlan version 3.0 (Brainlab) for contouring target volumes and organs at risk (OAR) and producing stereotactic RT plans. Gross tumor volume (GTV) and OAR were outlined on the MRI scans. Planning target volume (PTV) was the same as GTV without margin. Stereotactic RT plans were mostly composed of 3-6 arc beams, except for 2 patients who received intensity-modulated RT with 7-8 beams, and aimed to deliver $90 \%$ of the prescribed dose to the PTV. Stereotactic RT was performed using Novalis linear accelerator from single to 10 fractions under image-guidance using an ExacTrac device (Brainlab).

\section{Measurement of tumors}

Serial T1-weighted axial post-contrast MRI scans before and after stereotactic RT were uploaded to MIM 6.6 (MIM Software Inc., Cleveland, $\mathrm{OH}$, USA) to measure the longest diameters and volumes of VS. The VS outlines were drawn in the axial MRI scans, and the longest diameter was measured as the linear component of RECIST from the VS contours. Tumor volume (TV) was created after piling up the contours of VS in the axial MRI scans. VS are usually well enhanced because of extraaxial tumors, but some portions are less enhanced. Enhanced tumor volume (ETV) within a TV was defined as a hyperenhancing volume in a TV compared to normal tissues. The pons was selected as a reference normal tissue to get a cutoff of hyper-enhancement [9]. An axial MRI scan of the midlevel of the pons was contoured and a reference plane was produced. Mean signal intensity in the plane was used for the enhancement threshold for ETV (Fig. 1).

\section{Evaluation of size changes and responses}

Percent changes of the sizes of VS were produced by comparing follow-up MRI scans with pre-RT ones in terms of maximum diameters, TV, and ETV, respectively. Disappearance of VS was categorized as complete response (CR). Partial response (PR) and progressive disease (PD) were defined by RECIST as 30\% decrease and 20\% increase in the maximum diameter, respectively. To make the response criteria from the volumetric analysis correspond with RECIST, the VS shapes were supposed to be spherical. The cutoffs of PR and PD by volumetric measurements were 66\% reduction and $73 \%$ increase, respectively $[10,11]$. Neither $C R, P R$, nor PD indicated stable disease (SD).

\section{Statistical analyses}

Statistical analyses were performed using SPSS version 23.0 (IBM Inc., Armonk, NY, USA). To evaluate the correspondence of the measurement methods, we examined the correlation of size changes and the agreement of responses. The Pearson 

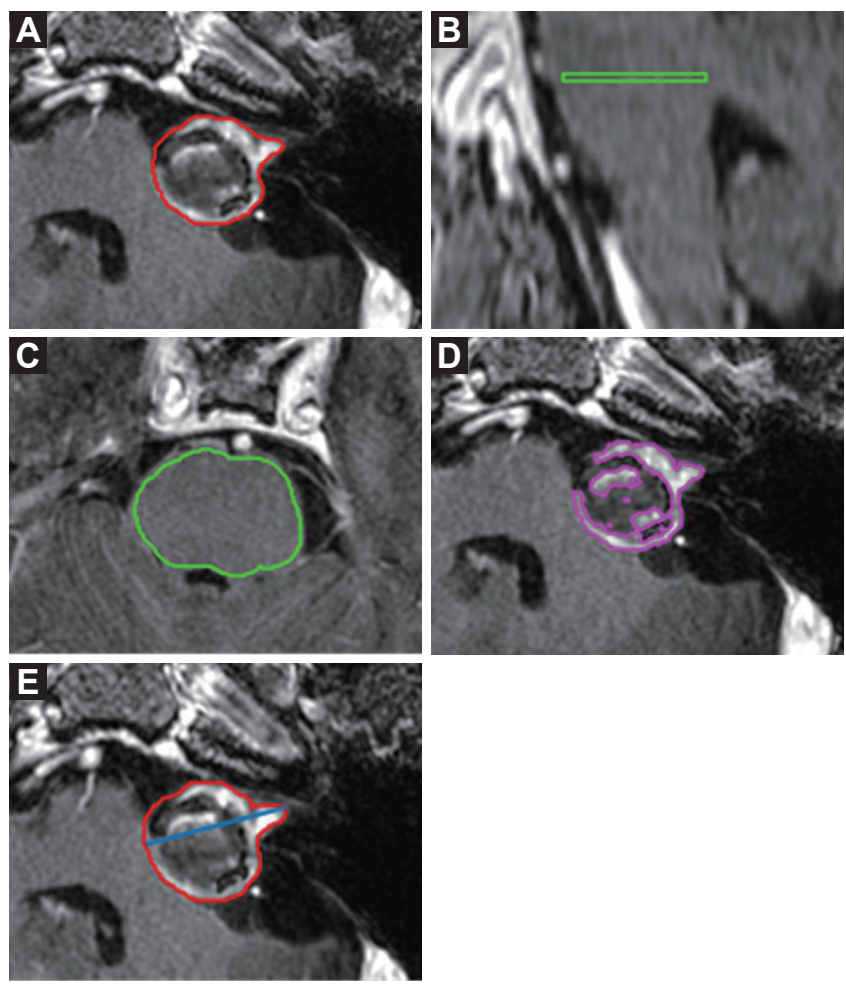

Fig. 1. Size measurement of a vestibular schwannoma (VS). Tumor volume was created after piling up the contours of VS in T1-weighted axial post-contrast magnetic resonance imaging (MRI) scans (A). Mean signal intensity of mid-level of the pons was selected as the cut-off of hyper-enhancement $(B, C)$, and an enhanced tumor volume was defined as the volume of signal intensity beyond it (D). Maximum diameter in an axial MRI scan was measured (E).

correlation coefficient was calculated to determine the concordance of the percent changes of VS sizes between pairs of the measurement methods. Cohen kappa statistics was carried out to evaluate the response agreement between the measurements in a pairwise fashion (Table 1). Local control rates were performed with Kaplan-Meier methods, and the influencing factors were analyzed with the log rank test.

\section{Results}

Among the 30 patients, 4 received RT after partial resection, and the others did so primarily. Three fractionations were most commonly applied to 14 patients, single fraction to 8 patients, and 4 to 10 fractions to the rest (Table 2). The median dose of RT was $21 \mathrm{~Gy}$ (range, 13.0 to $40.0 \mathrm{~Gy}$ ), and the median biologically effective dose (BED) using an $\alpha / \beta$ ratio of $2.47 \mathrm{~Gy}$ was $81.4 \mathrm{~Gy}$ (range, 60.5 to 107.4 Gy). Median follow-up from
Table 1. Interpretation of kappa values

\begin{tabular}{ll}
\hline \multicolumn{1}{c}{ Kappa } & \multicolumn{1}{c}{ Agreement } \\
\hline$<0$ & Less than chance agreement \\
$0.01-0.20$ & Slight agreement \\
$0.21-0.40$ & Fair agreement \\
$0.41-0.60$ & Moderate agreement \\
$0.61-0.80$ & Substantial agreement \\
$0.81-0.99$ & Almost perfect agreement \\
\hline
\end{tabular}

Table 2. Patient characteristics

\begin{tabular}{lc}
\hline \multicolumn{1}{c}{ Characteristic } & Value \\
\hline Age $(\mathrm{yr})$ & $58.5(24-79)$ \\
Sex & 13 \\
$\quad$ Male & 17 \\
Female & $1.7(0.9-4.1)$ \\
Longest diameter of initial tumors (cm) & $0.8(0.2-17.1)$ \\
Volume of initial tumors $\left(\mathrm{cm}^{3}\right)$ & \\
Aim of radiotherapy & 26 \\
Primary & 4 \\
Postoperative & $21(13-40)$ \\
Dose of radiotherapy (Gy) & \\
Fraction numbers of radiotherapy & 8 \\
1 & 14 \\
3 & 3 \\
4 & 3 \\
5 & 2 \\
\hline
\end{tabular}

Values are presented as median (range) or number.

the initial MRI scans to the latest ones was 31.0 months (range, 3.5 to 86.5 months). The last MRI scans were taken within a year in 5 patients; in 2 the scans were taken within 6 months.

\section{Size changes according to the measurement method}

Temporary enlargements followed by subsequent shrinkage were found in 11 (36.7\%), 17 (56.7\%), and 9 (30.0\%) among 30 VS according to maximum diameter, TV, and ETV, respectively (Fig. 2). The median period to peak enlargement was 7.5 months (range, 3 to 29 months), 7.0 months (range, 2.5 to 33.5 months), and 10.0 months (range, 2.5 to 33.5 months), respectively, after RT. The Percent change of VS size compared to the pre-RT condition was calculated in 90 sets of follow-up MRI scans. Pearson correlation coefficients showed very strong concordance between either maximum diameters and TV, or TV and ETV: as well as strong concordance between maximum diameters and ETV (Fig. 3). 

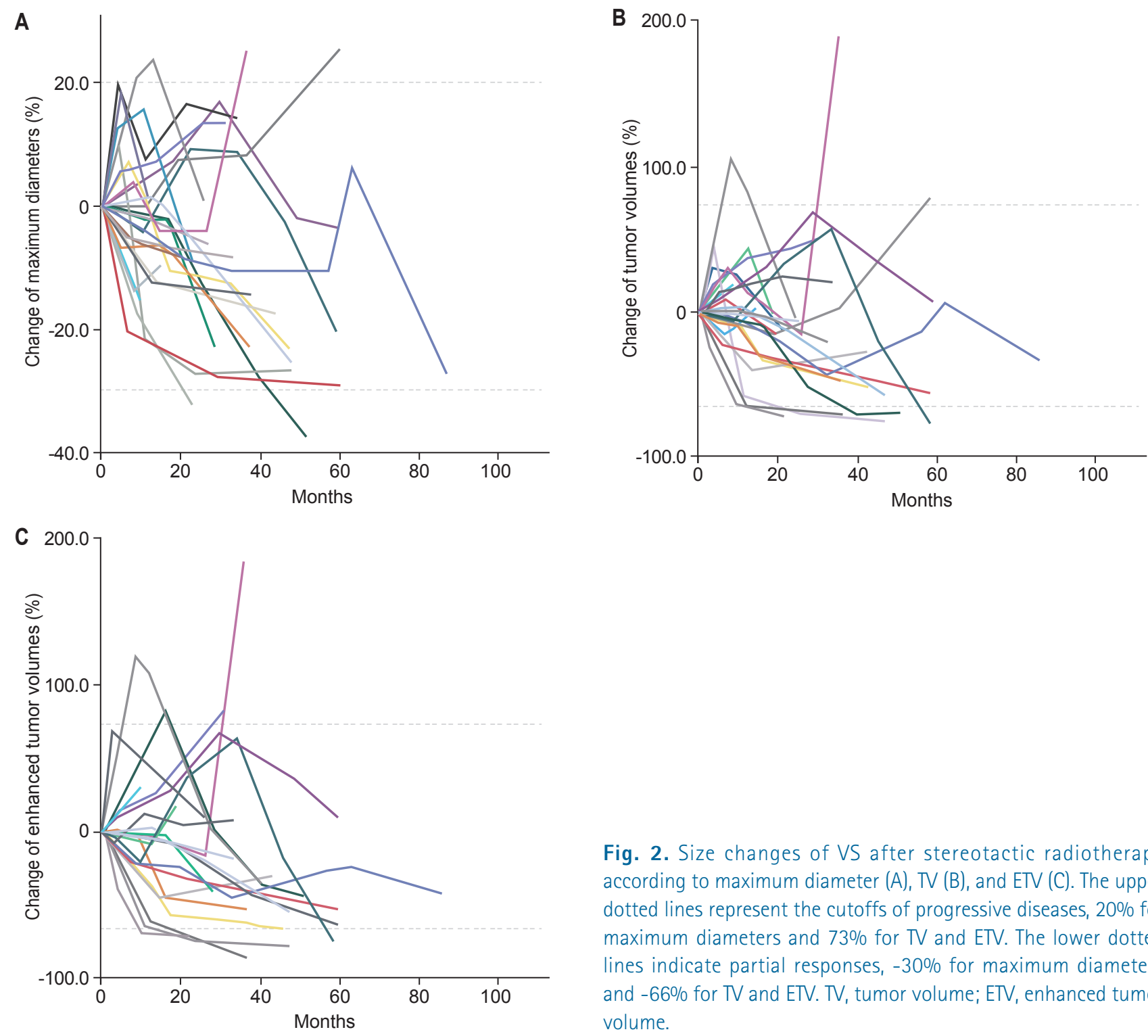

Fig. 2. Size changes of VS after stereotactic radiotherapy according to maximum diameter (A), TV (B), and ETV (C). The upper dotted lines represent the cutoffs of progressive diseases, 20\% for maximum diameters and 73\% for TV and ETV. The lower dotted lines indicate partial responses, -30\% for maximum diameters and -66\% for TV and ETV. TV, tumor volume; ETV, enhanced tumor volume.

Table 3. Agreement of responses among the measurement methods

\begin{tabular}{|c|c|c|c|c|c|c|}
\hline & \multicolumn{3}{|c|}{ Number of response } & \multicolumn{3}{|c|}{ Frequency of agreement } \\
\hline & Partial response & Stable disease & Progressive disease & RECIST & TV & ETV \\
\hline RECIST & $3(3.3)$ & 83 (92.2) & $4(4.4)$ & - & 83 (92.2) & 78 (86.7) \\
\hline TV & $7(7.8)$ & 79 (87.8) & $4(4.4)$ & & - & 83 (92.2) \\
\hline ETV & $7(7.8)$ & 78 (86.7) & $5(5.6)$ & & & - \\
\hline
\end{tabular}

Values are presented as number (\%).

RECIST, Response Evaluation Criteria in Solid Tumors; TV, tumor volume; ETV, enhanced tumor volume.

\section{Responses determined by measurement method}

The responses according to the measurement method used at each follow-up MRI scan are shown in Table 3. There was no complete remission by any assessment method, and SD was $\geq 86.7 \%$ by each method. Identical responses were found in $92.2 \%$ of either RECIST-TV or TV-ETV, and $86.7 \%$ of RECISTETV. Kappa values for the agreement of responses in RECISTTV, RECIST-ETV, and TV-ETV were 0.58 (95\% confidence interval 
A

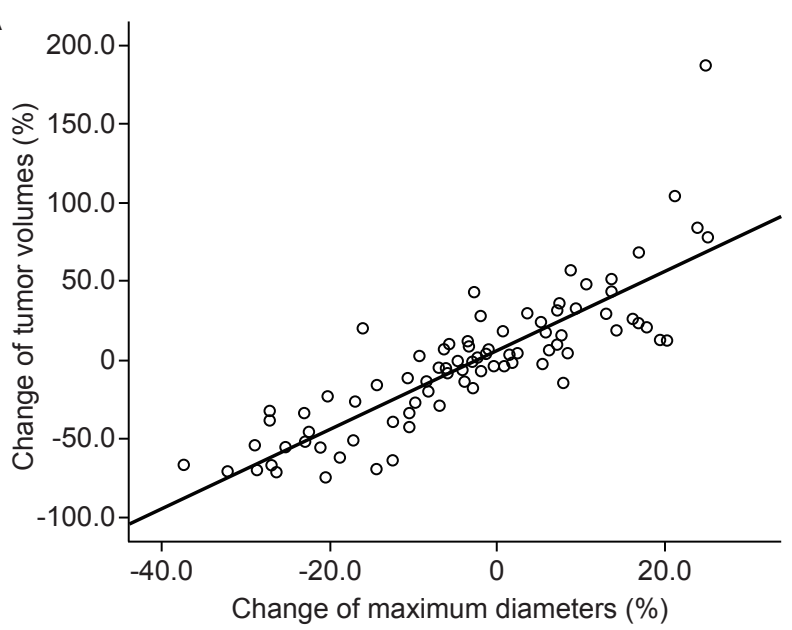

B
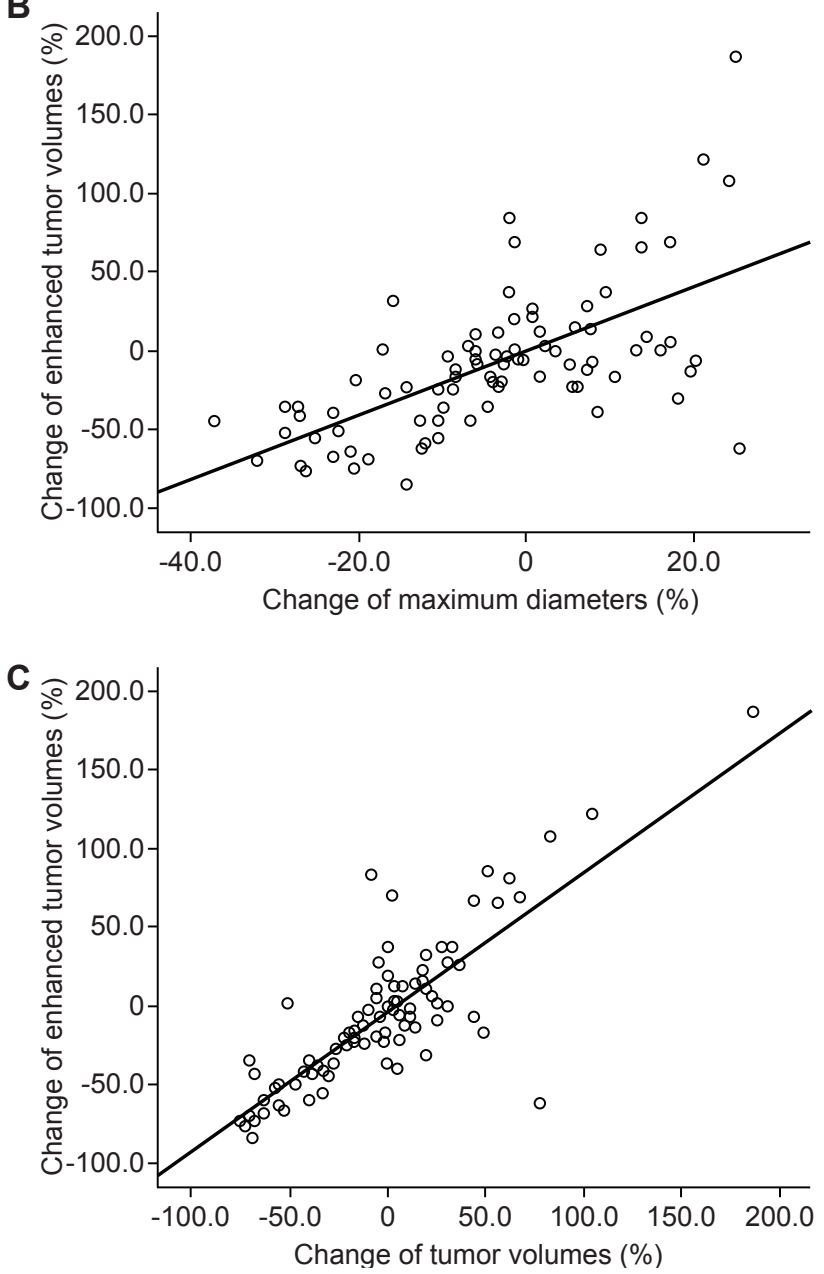

Fig. 3. Correlation of size changes among the measurement methods. Pearson correlation coefficients between (A) RECIST and TV, (B) RECIST and ETV, and (C) TV and ETV were 0.85 (95\% confidence intervals [Cl], 0.80-0.90), $0.63(95 \% \mathrm{Cl}, 0.47-0.76)$, and $0.82(95 \% \mathrm{Cl}, 0.64-0.92)$, respectively $(p=0.000)$. RECIST, Response Evaluation Criteria in Solid Tumors; TV, tumor volume; ETV, enhanced tumor volume.

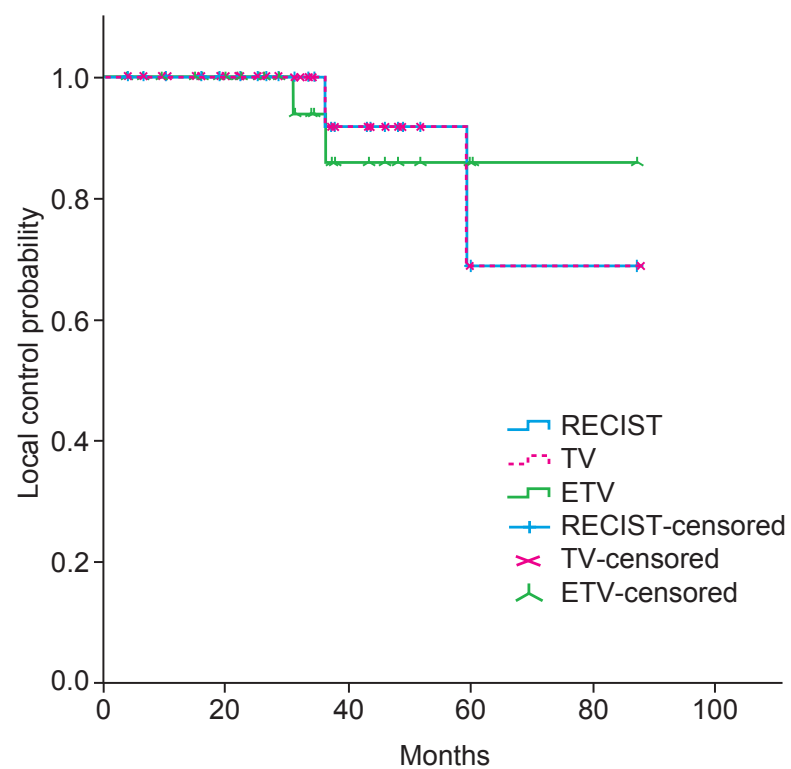

Fig. 4. Local control rates in vestibular schwannoma after stereotactic radiotherapy. RECIST, Response Evaluation Criteria in Solid Tumors; TV, tumor volume; ETV, enhanced tumor volume.

[Cl], 0.32-0.85), $0.32(95 \% \mathrm{Cl}, 0.05-0.59)$, and $0.66(95 \% \mathrm{Cl}$, $0.43-0.90)$, respectively $(p=0.000)$.

\section{Local control}

PDs were found in two VS from the last follow-up MRI scans by each measurement method. They occurred in the same VS according to RECIST and TV, but one of them did occur in a different VS according to ETV. Three- and 5-year local control rates were $91.7 \%$ and $68.8 \%$ with either RECIST or TV criteria, and $85.9 \%$ and $85.9 \%$ with ETV definitions, respectively (Fig. 4). Age, sex, initial tumor size, RT dose, BED, and fractionation did not influence the local control rates. Among four VS receiving postoperative RT, there was no PD. Three- and 5-year local control rates in VS receiving primary RT were $90.9 \%$ and $68.2 \%$ with either RECIST or TV criteria, and $92.9 \%$ and $84.4 \%$ with ETV definitions, respectively.

\section{Discussion and Conclusion}

The response measurement method in VS after RT has not yet determined, so the responses have been diverse according to studies done. Some applied RECIST using maximum diameters $[8,12]$, others volumes $[13,14]$. Although the volumetric measurement is accurate, it takes more effort and requires sophisticated computer systems compared to the linear maximum diameter. Therefore, we tried to evaluate 
the feasibility of the maximum diameter by analyzing the correspondence with the volumetric measurements. When there is a strong concordance of percent changes and designated responses between the linear and volumetric measurements, the maximum diameter can be used for the response measurement of VS after RT.

Percent changes of $V S$ receiving $R T$ were strongly concordant in pairwise comparisons between the linear and volumetric measurements in the present study (Fig. 3). Therefore, use of the maximum diameter can be considered a reasonable method for evaluating the size changes of VS after RT. The magnitude of percent changes was greater in TV and ETV compared to maximum diameters. These larger changes in volumetric measurements compared to the maximum linear dimensions were also reported in another study analyzing the percent changes of the growth rates of VS [10]. We think the difference was attributable to the difference of absolute values between volumetric measurements that were produced by triplicate linear components and just one-dimensional maximum diameters.

Transient enlargement followed by regression was reported in 30\%-57\% of VS receiving RT $[13,15-17]$ and did not develop beyond 2 years after RT $[14,15]$. In present analysis, the incidence of pseudo-progression found was from 30.3\% to $56.7 \%$ according to the measurement method, and the median interval of its peak enlargement ranged from 7 to 10 months after RT (range, 2.5 to 33.5 months). Therefore, the response evaluation of VS following $\mathrm{RT}$ requires a long follow-up period, especially in the case of an initially enlarged VS after RT, because the enlargement may be temporary.

In this study, RT responses were defined using the guidelines of one-dimensional RECIST and three-dimensional volumetric methods. In $86.7 \%$ or more cases, there was agreement between RECIST and the volumetric determinations (Table 3). Moreover, the kappa values between RECIST, and either TV or ETV, showed moderate or fair agreement, respectively. When the $95 \% \mathrm{Cl}$ for kappa statistics does not contain zero, this indicates statistically significant agreement between the two measurements at the 0.05 level (i.e., $p<0.05$ ) [18]. All the 95\% Cls in the kappa analyses were above zero in the present study, so the agreement between RECIST and the volumetric judgements was significant.

Three- and 5-year local control rates were 91.7\% and 68.8\% by RECIST or TV, and $85.9 \%$ and $85.9 \%$ by ETV, respectively (Fig. 4). The local control rates in this study were lower than those in other studies (about 90\%) [4-6]. There are several possible explanations. First, the number of VS patients was small in this study. When analyzing data of low event incidence, such as finding a $90 \%$ control rate, the control rates can be greatly influenced by a small change of the incidence. Therefore, studies with a larger sample size are required. Second, the follow-up period was too short to calculate the 5-year local control. Temporary enlargement was found in $1 / 3$ to $1 / 2$ of cases, according to the measurements in the present analysis, so the follow-up periods beyond this are required for an adequate response evaluation.

There were two PDs that disagreed among the measurement methods. One VS was determined as PD only by ETV. It enlarged consistently according to all the measurement methods after RT, until the last follow-up at 30.5 months. For VS having a trend of enlargement like this, a follow-up period must be sufficiently long to verify whether it is true PD or pseudo-progression. The other was defined as PD by RECIST and TV, but not by ETV. It also increased in size, but had a large proportion of central necrosis in the last follow-up MRI scans at 59.0 months. Therefore, PD by RECIST or TV may need further evaluation with enhanced MRI scans to identify whether there is a large portion of central necrosis.

Loss of central enhancement was observed in VS after RT $[13,16,19,20]$, but its clinical significance, especially correlation with tumor shrinkage, has been inconclusive. Schneider et al. found that the percentage of enhancing VS volume was nearly constant after gamma knife radiosurgery, but continuously dropped after fractionated stereotactic RT [9]. Scheller et al. [20] did not found necrotic tumor areas in the operative specimens which had been considered as necrotic tumor areas on MRI. So, they insisted that loss of contrast uptake in previously irradiated VS may not be indicative of effective radiotherapy. Therefore, in order to elucidate the clinical significance of ETV, prospective studies are necessary.

Four VS received postoperative RT in this study. Because of the small patient number, it was difficult to compare the percent change, response, and local control in VS according to either postoperative or primary RT. Although local control rates without them was similar to entire patients, we think the effect of postoperative RT is out of scope in the present analysis. Therefore, meticulous studies about them are required in the future.

While RECIST defines more than 20\% increase as progression, volumetric measurements of VS after RT do not provide a consensus on progression. The definitions of volumetric progression were 10\% to 73\% different according to different studies $[10,11,13,14,17]$. VS responds much slower than malignant tumors do after RT, so VS may require a 
different cut-off of volumetric progression. Because of the low incidence and slow response of VS, multicenter studies involving large patient number are required to make a clinically valuable definition of its volumetric progression after RT.

The single maximum diameter has commonly been used to estimate the growth of VS $[8,21]$, but its limitations were also reported. Vokurka et al. [22] found that a maximal diameter measurement showed poorer correlation with manual segmented volumes compared to Bayesian partial volume segmentation, and insisted that it did not provide adequate statistical accuracy to monitor tumor growth. Varughese et al. [23] found that maximum diameter had greater re-test errors compared to area-based measurements, and suggested that it should not be used as an approximation method. The conflicts about the effectiveness of RECIST in VS highlight the caution needed when using RECIST.

This study carries unavoidable weaknesses of retrospective nature, including small sample size, short follow-up time, heterogeneous RT fractionations, and different periods and slice thicknesses of follow-up MRI scans. Nevertheless, the strong concordance of size change and significant agreement of responses between the one- and three-dimensional measurements in the present study support the practical feasibility of using the maximum diameter and RECIST in the response assessment of VS following RT.

In conclusion, one-dimensional measurement, such as maximum diameter and RECIST, instead of the threedimensional volumetric ones, can be used for the evaluation of VS response after RT, assuming a follow-up period adequate to evaluate the cases of temporary enlargement.

\section{Conflict of Interest}

No potential conflict of interest relevant to this article was reported.

\section{Acknowledgments}

This work was supported by the Dong-A University research fund.

\section{References}

1. Stepanidis K, Kessel M, Caye-Thomasen P, Stangerup SE. Socio-demographic distribution of vestibular schwannomas in Denmark. Acta Otolaryngol 2014;134:551-6.

2. Carlson ML, Habermann EB, Wagie $A E$, et al. The changing landscape of vestibular schwannoma management in the United States: a shift toward conservatism. Otolaryngol Head Neck Surg. 2015;153:440-6.

3. Kleijwegt M, Ho V, Visser O, Godefroy W, van der Mey A. Real incidence of vestibular schwannoma? Estimations from a national registry. Otol Neurotol 2016;37:1411-7.

4. Hansasuta A, Choi CY, Gibbs IC, et al. Multisession stereotactic radiosurgery for vestibular schwannomas: single-institution experience with 383 cases. Neurosurgery 2011;69:1200-9.

5. Champ CE, Shen $X$, Shi W, et al. Reduced-dose fractionated stereotactic radiotherapy for acoustic neuromas: maintenance of tumor control with improved hearing preservation. Neurosurgery 2013;73:489-96.

6. Hasegawa T, Kato T, Kida Y, et al. Gamma Knife surgery for patients with jugular foramen schwannomas: a multiinstitutional retrospective study in Japan. J Neurosurg 2016;125:822-31.

7. Puataweepong P, Dhanachai M, Dangprasert S, et al. Linacbased stereotactic radiosurgery and fractionated stereotactic radiotherapy for vestibular schwannomas: comparative observations of 139 patients treated at a single institution. J Radiat Res 2014;55:351-8.

8. Combs SE, Engelhard C, Kopp C, et al. Long-term outcome after highly advanced single-dose or fractionated radiotherapy in patients with vestibular schwannomas: pooled results from 3 large German centers. Radiother Oncol 2015;114:378-83.

9. Schneider T, Chapiro J, Lin M, et al. 3D quantitative assessment of response to fractionated stereotactic radiotherapy and single-session stereotactic radiosurgery of vestibular schwannoma. Eur Radiol 2016;26:849-57.

10. Walz PC, Bush ML, Robinett Z, Kirsch CF, Welling DB. Threedimensional segmented volumetric analysis of sporadic vestibular schwannomas: comparison of segmented and linear measurements. Otolaryngol Head Neck Surg 2012;147:737-43.

11. Dombi E, Ardern-Holmes SL, Babovic-Vuksanovic D, et al. Recommendations for imaging tumor response in neurofibromatosis clinical trials. Neurology 2013;81(21 Suppl 1):S33-40.

12. Woolf DK, Williams $M$, Goh $C L$, et al. Fractionated stereotactic radiotherapy for acoustic neuromas: long-term outcomes. Clin Oncol (R Coll Radiol) 2013;25:734-8.

13. Okunaga $T$, Matsuo $T$, Hayashi $N$, et al. Linear accelerator radiosurgery for vestibular schwannoma: measuring tumor volume changes on serial three-dimensional spoiled gradientecho magnetic resonance images. J Neurosurg 2005;103:53-8.

14. Hayhurst C, Zadeh G. Tumor pseudoprogression following radiosurgery for vestibular schwannoma. Neuro Oncol 
2012;14:87-92.

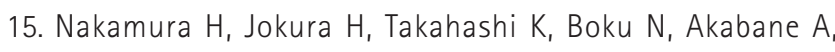
Yoshimoto T. Serial follow-up MR imaging after gamma knife radiosurgery for vestibular schwannoma. AJNR Am J Neuroradiol 2000;21:1540-6.

16. Pollock BE. Management of vestibular schwannomas that enlarge after stereotactic radiosurgery: treatment recommendations based on a 15 year experience. Neurosurgery 2006;58:241-8.

17. Meijer OW, Weijmans EJ, Knol DL, et al. Tumor-volume changes after radiosurgery for vestibular schwannoma: implications for follow-up MR imaging protocol. AJNR Am J Neuroradiol 2008;29:906-10.

18. Galanis E, Buckner JC, Maurer MJ, et al. Validation of neuroradiologic response assessment in gliomas: measurement by RECIST, two-dimensional, computer-assisted tumor area, and computer-assisted tumor volume methods. Neuro Oncol 2006;8:156-65.

19. Andrews DW, Suarez O, Goldman HW, et al. Stereotactic radiosurgery and fractionated stereotactic radiotherapy for the treatment of acoustic schwannomas: comparative observations of 125 patients treated at one institution. Int J Radiat Oncol Biol Phys 2001;50:1265-78.

20. Scheller C, Rampp S, Rachinger JC, et al. Contrast enhancement and histopathological findings in vestibular schwannoma. Cent Eur Neurosurg 2010;71:35-8.

21. Kim CH, Chung KW, Kong DS, et al. Prognostic factors of hearing preservation after gamma knife radiosurgery for vestibular schwannoma. J Clin Neurosci 2010;17:214-8.

22. Vokurka EA, Herwadkar A, Thacker NA, Ramsden RT, Jackson A. Using Bayesian tissue classification to improve the accuracy of vestibular schwannoma volume and growth measurement. AJNR Am J Neuroradiol 2002;23:459-67.

23. Varughese JK, Wentzel-Larsen T, Vassbotn F, Moen G, LundJohansen M. Analysis of vestibular schwannoma size in multiple dimensions: a comparative cohort study of different measurement techniques. Clin Otolaryngol 2010;35:97-103. 